\title{
Well-posed results for nonlocal biparabolic equation with linear and nonlinear source terms
}

\author{
Le Dinh Long ${ }^{1}$, Ho Duy Binh ${ }^{1}$, Kim Van Ho Thi ${ }^{1}$ and Van Thinh Nguyen ${ }^{2^{*}}$ (D)
}

\section{"Correspondence:}

vnguyen@snu.ac.kr

${ }^{2}$ Department of Civil and

Environmental Engineering, Seoul

National University, Seoul, South Korea

Full list of author information is available at the end of the article

\begin{abstract}
In this paper, we consider the biparabolic problem under nonlocal conditions with both linear and nonlinear source terms. We derive the regularity property of the mild solution for the linear source term while we apply the Banach fixed-point theorem to study the existence and uniqueness of the mild solution for the nonlinear source term. In both cases, we show that the mild solution of our problem converges to the solution of an initial value problem as the parameter epsilon tends to zero. The novelty in our study can be considered as one of the first results on biparabolic equations with nonlocal conditions.
\end{abstract}

Keywords: Biparabolic equation; Source term; Nonlocal condition; Mild solution; Existence; Uniqueness; Convergence

\section{Introduction}

Let $\Omega$ be a bounded domain in $\mathbb{R}^{N}(N \geq 1)$ with sufficiently smooth boundary $\partial \Omega$. In this paper, we consider the following biparabolic equation:

$$
\begin{cases}u_{t t}(x, t)+2 \Delta u_{t}(x, t)+\Delta^{2} u(x, t)=F(u(x, t)) & \text { in } \Omega \times(0, T] \\ \left.u\right|_{\partial \Omega}=\left.\Delta u\right|_{\partial \Omega}=0 & \text { in } \Omega, \\ u_{t}(x, 0)=0 & \text { in } \partial \Omega,\end{cases}
$$

under temporal nonlocal condition

$$
u(x, 0)+\varepsilon u(x, T)=f(x), \quad x \in \partial \Omega .
$$

Here $u(x, t)$ is a function of temperature or concentration, $F(u)$ is a source function, $\varepsilon$ is a parameter, and $f \in L^{2}(\Omega) \cap \mathbb{H}^{s}(\Omega)$. When $\varepsilon=0$, the problem becomes an initial conditional problem.

The main equation of problem (1.1) is equivalent to

$$
P^{2} u=P(P u)=\frac{\partial^{2}}{\partial t^{2}} u+2 \frac{\partial}{\partial t} \mathcal{A} u+\mathcal{A}^{2} u=G(x, t ; u),
$$

(c) The Author(s) 2021. This article is licensed under a Creative Commons Attribution 4.0 International License, which permits use sharing, adaptation, distribution and reproduction in any medium or format, as long as you give appropriate credit to the original author(s) and the source, provide a link to the Creative Commons licence, and indicate if changes were made. The images or other third party material in this article are included in the article's Creative Commons licence, unless indicated otherwise in a credit line to the material. If material is not included in the article's Creative Commons licence and your intended use is not permitted by statutory regulation or exceeds the permitted use, you will need to obtain permission directly from the copyright holder. To view a copy of this licence, visit http://creativecommons.org/licenses/by/4.0/. 
where $P$ is the main operator for the classical parabolic equation,

$$
P u=\left(\frac{\partial}{\partial t}+\mathcal{A}\right) u=G(x, t ; u)
$$

As mentioned by Fushchich, Galitsyn, and Polubinskii [9], the new fourth-order partial differential equation (1.1) is invariant with respect to the Galilei group. From the results in [9] we realize that the classical heat equations

$$
u_{t}-\Delta u=0
$$

do not completely describe heat and mass transfer processes. Therefore, in many situations of heat conduction, it tends to replace the classical thermal equation by one of the hyperbolic form, such as problem (1.1). Problem (1.1) is a form of quadratic PDEs equations, which have a wide range of applications in various scientific and engineering disciplines, such as conduction of heat $[7,9,24,33]$, dynamics of filtration consolidation [6, 8], strongly damped wave equations [14, 23, 34], ice formulation and accretion problems on structures, ships, and aircraft [19-21], the transport of liquids and insoluble surfactant through the lung airways [11, 12], brain imaging for the detection and mapping of subtle abnormalities of shape and volume in the brains of patients with metastatic tumors $[18,26,27]$, and so on.

Whereas there were a number of studies focused on parabolic equations $[1-4,10,13,15$, $22,25,28]$, the studies on biparabolic equations are still limited. Let us mention previous works related to biparabolic equation (1.1). Lakhdari and Boussetila [16] applied KozlovMaz'ya iteration method for approximating the final value problem for biparabolic equation. Bulavatsky [7] studied some boundary value problems for biparabolic equations with nonlocal boundary conditions. Besma et al. [5] considered the problem of approximating a solution of an ill-posed biparabolic problem in the abstract Hilbert space. They introduced a modified quasi-boundary value method to get stable solutions for regularizing the ill-posedness of a biparabolic equation. Tuan et al. [32] studied the problem of finding the initial distribution for a linear inhomogeneous or nonlinear biparabolic equation. Recently, Phuong et al. [25] studied an inverse source problem of the biparabolic equation. Very recently, Tuan et al. [31] investigated two terminal value problems for stochastic biparabolic equations perturbed by a standard Brownian motion or a fractional Brownian motion.

The nonlocal problem focused in this paper is considered as one of the most interesting areas for the readers in various applications, such as chaos, chemistry, biology, and physics; see [30]. In comparison with the initial or final conditions, the nonlocal conditions are more difficult to handle. The novelty of our problem is the presence of condition of nonlocal type (1.2). In many real-world applications, it is difficult to collect accurate data at the beginning or at the end of a process. In addition, many processes happen so fast and in a short period, in which we only can observe the data at the beginning and the end of a process, not the data at a specific time in the range of $(0, T)$. Therefore studies on nonlocal conditional problems can help us to track down a process in more detail and in an effective way. 
To the best of our knowledge, up to date, there is still no any study considering problem (1.1) under the nonlocal condition (1.2). This motivates us to focus on problems (1.1)(1.2). The main contributions of the paper are as follows.

- For the linear source function, we give the well-posedness and investigate the convergence of the mild solution to problem (1.1)-(1.2) as $\varepsilon$ approaches 0 . In more detail, we prove that the solution of problem (1.1)-(1.2) converges to a mild solution with the initial value problem for (1.1).

- For nonlinear source functions, we prove the existence and uniqueness of mild solutions. In the main analysis, we apply the Banach fixed point theorem. Our next aim is to demonstrate the convergence of the mild solution as the parameter $\varepsilon$ tends to 0 .

The main techniques to handle the above problem are based on the ideas of some recent publications $[17,29,30]$. We overcome some difficulties by setting up complex evaluations on Hilbert scale spaces. Choosing the right spaces for the input $f$ and for the solution is also not simple task.

This paper is organized as follows. In Sect. 2, we provide some useful notations and the definition of a solution in the mild sense. In Sect. 3, we focus on the well-posed results for the linear case and discuss on what happens as $\varepsilon \rightarrow 0$. The well-posed results for the nonlinear source term are introduced in Sect. 4. Eventually, the results are summarized in Sect. 5 .

\section{Preliminary results and mild solution}

In this section, we introduce the notation and the functional setting used in our paper. Recall that the spectral problem

$$
\begin{cases}\Delta \psi_{n}(x)=-\lambda_{n} \psi_{n}(x), & x \in \Omega, \\ \psi_{n}(x)=0, & x \in \partial \Omega,\end{cases}
$$

admits eigenvalues $0<\lambda_{1} \leq \lambda_{2} \leq \cdots \leq \lambda_{n} \leq \cdots$ with $\lambda_{n} \rightarrow \infty$ as $n \rightarrow \infty$. The corresponding eigenfunctions are $\psi_{n} \in H_{0}^{1}(\Omega)$.

Definition 2.1 (Hilbert scale space) We recall the Hilbert scale space given as follows:

$$
\mathbb{H}^{s}(\Omega)=\left\{f \in L^{2}(\Omega), \sum_{n=1}^{\infty} \lambda_{n}^{2 s}\left(\int_{\Omega} f(x) \psi_{n}(x) d x\right)^{2}<\infty\right\}
$$

for $s \geq 0$. It is well known that $\mathbb{H}^{s}(\Omega)$ is the Hilbert space corresponding to the norm

$$
\|f\|_{\mathbb{H}^{s}(\Omega)}=\left(\sum_{j=1}^{\infty} \lambda_{j}^{2 s}\left(\int_{\Omega} f(x) \psi_{n}(x) d x\right)^{2}\right)^{1 / 2}, \quad f \in \mathbb{H}^{s}(\Omega) .
$$

Let us give an explicit formula of the mild solution. First, taking the inner product of both sides of (1.1) with $\psi_{n}(x)$, we find that

$$
\begin{aligned}
& \frac{d^{2}}{d t^{2}}\left(\int_{\Omega} u(x, t) \psi_{n}(x) d x\right)+2 \lambda_{n}\left(\int_{\Omega} u(x, t) \psi_{n}(x) d x\right) \\
& \quad+\lambda_{n}^{2}\left(\int_{\Omega} u(x, t) \psi_{n}(x) d x\right)=\int_{\Omega} F(u(x, t)) \psi_{n}(x) d x .
\end{aligned}
$$


It is easy to see that the latter problem has a solution given by

$$
\begin{aligned}
\int_{\Omega} u(x, t) \psi_{n}(x) d x= & e^{-t \lambda_{n}}\left(1+t \lambda_{n}\right) \int_{\Omega} u(x, 0) \psi_{n}(x) d x \\
& +\int_{0}^{t}(t-r) e^{-(t-r) \lambda_{n}}\left(\int_{\Omega} F(u(x, r)) \psi_{n}(x) d x\right) d r .
\end{aligned}
$$

The condition $u(x, 0)+\varepsilon u(x, T)=f(x)$ implies that

$$
\begin{aligned}
& \int_{\Omega} u(x, 0) \psi_{n}(x) d x+\varepsilon \int_{\Omega} u(x, T) \psi_{n}(x) d x \\
& =\left(1+\varepsilon e^{-T \lambda_{n}}\left(1+T \lambda_{n}\right)\right) \int_{\Omega} u(x, 0) \psi_{n}(x) d x \\
& \quad+\varepsilon \int_{0}^{T}(T-r) e^{-(T-r) \lambda_{n}}\left(\int_{\Omega} F(u(x, r)) \psi_{n}(x) d x\right) d r \\
& =\int_{\Omega} f(x) \psi_{n}(x) d x .
\end{aligned}
$$

We rewrite it as

$$
\begin{aligned}
& \int_{\Omega} u(x, 0) \psi_{n}(x) d x \\
& \quad=\frac{\int_{\Omega} f(x) \psi_{n}(x) d x-\varepsilon \int_{0}^{T}(T-r) e^{-(T-r) \lambda_{n}}\left(\int_{\Omega} F(u(x, r)) \psi_{n}(x) d x\right) d r}{1+\varepsilon e^{-T \lambda_{n}}\left(1+T \lambda_{n}\right)} .
\end{aligned}
$$

Combining (2.2) and (2.4), we find that

$$
\begin{aligned}
\int_{\Omega} u(x, t) \psi_{n}(x) d x= & \frac{e^{-t \lambda_{n}}\left(1+t \lambda_{n}\right)}{1+\varepsilon e^{-T \lambda_{n}}\left(1+T \lambda_{n}\right)} \int_{\Omega} f(x) \psi_{n}(x) d x \\
& -\frac{\varepsilon e^{-t \lambda_{n}}\left(1+t \lambda_{n}\right) \int_{0}^{T}(T-r) e^{-(T-r) \lambda_{n}}\left(\int_{\Omega} F(u(x, r)) \psi_{n}(x) d x\right) d r}{1+\varepsilon e^{-T \lambda_{n}}\left(1+T \lambda_{n}\right)} \\
& +\int_{0}^{t}(t-r) e^{-(t-r) \lambda_{n}}\left(\int_{\Omega} F(u(x, r)) \psi_{n}(x) d x\right) d r .
\end{aligned}
$$

For any $f \in L^{2}(\Omega)$, we define

$$
\mathbf{Q}_{\varepsilon}(t) f=\sum_{n=1}^{\infty} \frac{e^{-t \lambda_{n}}\left(1+t \lambda_{n}\right)}{1+\varepsilon e^{-T \lambda_{n}}\left(1+T \lambda_{n}\right)}\left(\int_{\Omega} f(x) \psi_{n}(x) d x\right) \psi_{n}(x)
$$

and

$$
\mathbf{S}(t) f=e^{-t \Delta} f=\sum_{n=1}^{\infty} e^{-t \lambda_{n}}\left(\int_{\Omega} f(x) \psi_{n}(x) d x\right) \psi_{n}(x)
$$


From (2.5) we give an explicit formula of the solution to problem (1.1)-(1.2) in the mild setting:

$$
\begin{aligned}
u_{\varepsilon}(t)= & \mathbf{Q}_{\varepsilon}(t) f+\int_{0}^{t}(t-r) \mathbf{S}(t-r) F\left(u_{\varepsilon}(r)\right) d r \\
& -\varepsilon \mathbf{Q}_{\varepsilon}(t) \int_{0}^{T}(T-r) \mathbf{S}(T-r) F\left(u_{\varepsilon}(r)\right) d r .
\end{aligned}
$$

\section{Well-posed results for linear case}

In this section, we focus on the case $F(t, u)=F(t)$. Under the linear case, we recall the mild solution $u_{\varepsilon}$ to problem (1.1)-(1.2):

$$
u_{\varepsilon}(t)=\mathbf{Q}_{\varepsilon}(t) f+\int_{0}^{t}(t-r) \mathbf{S}(t-r) F(r) d r-\varepsilon \mathbf{Q}_{\varepsilon}(t) \int_{0}^{T}(T-r) \mathbf{S}(T-r) F(r) d r .
$$

Lemma 3.1 Let $f \in \mathbb{H}^{s}(\Omega)$.

a) If $s<m+1$, then

$$
\left\|\mathbf{Q}_{\varepsilon}(t) f\right\|_{\mathbb{H}^{m}(\Omega)} \leq C(s, m) t^{s-m-1}\|f\|_{\mathbb{H}^{s}(\Omega)} .
$$

b) If $s<m$, then

$$
\|\mathbf{S}(t) f\|_{\mathbb{H}^{m}(\Omega)} \leq C(s, m) t^{s-m}\|f\|_{\mathbb{H}^{s}(\Omega)} .
$$

Proof Using Parseval's equality, we find that

$$
\begin{aligned}
\|\mathbf{Q}(t) f\|_{\mathbb{H}^{m}(\Omega)}^{2} & =\sum_{n=1}^{\infty} \lambda_{n}^{2 m}\left(\frac{e^{-t \lambda_{n}}\left(1+t \lambda_{n}\right)}{1+\varepsilon e^{-T \lambda_{n}}\left(1+T \lambda_{n}\right)}\right)^{2}\left(\int_{\Omega} f(x) \psi_{n}(x) d x\right)^{2} \\
& \leq 2 \sum_{n=1}^{\infty} \lambda_{n}^{2 m} e^{-2 t \lambda_{n}}\left(1+t^{2} \lambda_{n}^{2}\right)\left(\int_{\Omega} f(x) \psi_{n}(x) d x\right)^{2} \\
& \leq 2 C_{T} \sum_{n=1}^{\infty} \lambda_{n}^{2 m+2} e^{-2 t \lambda_{n}}\left(\int_{\Omega} f(x) \psi_{n}(x) d x\right)^{2} .
\end{aligned}
$$

In view of the inequality $e^{-z} \leq C_{v} z^{-v}$ for all $v>0$, we know that

$$
\lambda_{n}^{2 m+2} e^{-2 t \lambda_{n}} \leq C_{v} \lambda_{n}^{2 m+2}\left(t \lambda_{n}\right)^{-2 v}=t^{-2 v} \lambda_{n}^{2 m+2-2 v} .
$$

It follows from (3.4) that

$$
\left\|\mathbf{Q}_{\varepsilon}(t) f\right\|_{\mathbb{H}^{m}(\Omega)}^{2} \leq t^{-2 v} \sum_{n=1}^{\infty} \lambda_{n}^{2 m+2-2 v}\left(\int_{\Omega} f(x) \psi_{n}(x) d x\right)^{2},
$$

which gives the estimate

$$
\left\|\mathbf{Q}_{\varepsilon}(t) f\right\|_{\mathbb{H}^{m}(\Omega)} \leq C_{T} t^{-v}\|f\|_{\mathbb{H}^{m+1-v}(\Omega)} .
$$


Setting $v=m+1-s>0$, we know that

$$
\left\|\mathbf{Q}_{\varepsilon}(t) f\right\|_{\mathbb{H}^{m}(\Omega)} \leq C(m, s) t^{s-m-1}\|f\|_{\mathbb{H}^{s}(\Omega)} .
$$

Using again $e^{-z} \leq C_{v} z^{-v}$ for all $v>0$, we find that

$$
\begin{aligned}
\|\mathbf{S}(t) f\|_{\mathbb{H}^{m}(\Omega)}^{2}=\left\|e^{-t \Delta} f\right\|_{\mathbb{H}^{m}(\Omega)}^{2} & =\sum_{n=1}^{\infty} \lambda_{n}^{2 m} e^{-2 t \lambda_{n}}\left(\int_{\Omega} f(x) \psi_{n}(x) d x\right)^{2} \\
& \leq C_{v} t^{-2 v} \lambda_{n}^{2 m-2 v}\left(\int_{\Omega} f(x) \psi_{n}(x) d x\right)^{2} \\
& =C_{v} t^{-2 v}\|f\|_{\mathbb{H}^{m-v}(\Omega)}^{2} .
\end{aligned}
$$

Setting $v=m-s$ for $s<m$, we get

$$
\|\mathbf{S}(t) f\|_{\mathbb{H}^{m}(\Omega)} \leq C(s, m) t^{s-m}\|f\|_{\mathbb{H}^{s}(\Omega)} .
$$

Theorem 3.1 Let $F \in L^{\infty}\left(0, T ; \mathbb{H}^{s-1}(\Omega)\right)$ and $f \in \mathbb{H}^{s}(\Omega)$. Then

$$
\left\|u_{\varepsilon}\right\|_{L^{\mu}\left(0, T ; \mathbb{H}^{m}(\Omega)\right)} \leq C(T, s, m, \mu)\left(\|f\|_{\mathbb{H}^{s}(\Omega)}+\|F\|_{L^{\infty}\left(0, T ; \mathbb{H}^{s-1}(\Omega)\right)}\right) .
$$

Proof Applying Lemma 3.1 and noting that $m<s<m+1$, we find that

$$
\begin{aligned}
\left\|u_{\varepsilon}(\cdot, t)\right\|_{\mathbb{H}^{m}(\Omega)} \leq & \left\|\mathbf{Q}_{\varepsilon}(t) f\right\|_{\mathbb{H}^{m}(\Omega)}+\int_{0}^{t}(t-r)\|\mathbf{S}(t-r) F(r)\|_{\mathbb{H}^{m}(\Omega)} d r \\
& +\varepsilon\left\|\mathbf{Q}_{\varepsilon}(t) \int_{0}^{T}(T-r) \mathbf{S}(T-r) F(r) d r\right\|_{\mathbb{H}^{m}(\Omega)} \\
\leq & C(m, s) t^{s-m-1}\|f\|_{\mathbb{H}^{s}(\Omega)}+\int_{0}^{t}(t-r)^{s-m}\|F(r)\|_{\mathbb{H}^{s-1}(\Omega)} d r \\
& +\varepsilon t^{s-m-1} \int_{0}^{T}(T-r)\|\mathbf{S}(T-r) F(r)\|_{\mathbb{H}^{s}(\Omega)} d r \\
= & I_{1}(t)+I_{2}(t)+I_{3}(t) .
\end{aligned}
$$

Let $\mu$ be such that $1<\mu<\frac{1}{m+1-s}$. The first term $I_{1}$ is bounded by

$$
\begin{aligned}
\left\|I_{1}\right\|_{L^{\mu}\left(0, T ; \mathbb{H}^{m}(\Omega)\right)} & \leq\left(\int_{0}^{T}\left|C(m, s) t^{s-m-1}\|f\|_{\mathbb{H}^{s}(\Omega)}\right|^{\mu} d t\right)^{1 / \mu} \\
& =\left(\frac{T^{(s-m-1) \mu+1}}{(s-m-1) \mu+1}\right)^{1 / \mu} C(m, s)\|f\|_{\mathbb{H}^{s}(\Omega)}
\end{aligned}
$$

For the second term $I_{2}$, we easily observe that

$$
I_{2}(t) \leq\left(\int_{0}^{t}(t-r)^{s-m} d r\right)\|F\|_{L^{\infty}\left(0, T ; \mathbb{H}^{s-1}(\Omega)\right)}=\frac{T^{s-m+1}}{s-m+1}\|F\|_{L^{\infty}\left(0, T ; \mathbb{H}^{s-1}(\Omega)\right)}
$$


Then we get the bound

$$
\begin{aligned}
\left\|I_{2}\right\|_{L^{\mu}\left(0, T ; \mathbb{H}^{m}(\Omega)\right)} & \leq\left(\int_{0}^{T}\left|\frac{T^{s-m+1}}{s-m+1}\|F\|_{L^{\infty}\left(0, T ; \mathbb{H}^{s-1}(\Omega)\right)}\right|^{\mu} d t\right)^{1 / \mu} \\
& =\frac{T^{s-m+1}}{s-m+1+1 \mu}\|F\|_{L^{\infty}\left(0, T ; \mathbb{H}^{s-1}(\Omega)\right)} .
\end{aligned}
$$

For the third term $I_{3}$, using Lemma (3.1), we have that

$$
\begin{aligned}
I_{3} & \leq \varepsilon t^{s-m-1} \int_{0}^{T}(T-r)\|\mathbf{S}(T-r) F(r)\|_{\mathbb{H}^{s}(\Omega)} d r \\
& \leq \varepsilon C(m, s) t^{s-m-1} \int_{0}^{T}(T-r)(T-r)^{-1}\|F(r)\|_{\mathbb{H}^{S-1}(\Omega)} d r \\
& \leq \varepsilon C(m, s) T\|F\|_{L^{\infty}\left(0, T ; \mathbb{H}^{s-1}(\Omega)\right)^{s-m-1}} .
\end{aligned}
$$

This immediately implies that

$$
\begin{aligned}
\left\|I_{3}\right\|_{L^{\mu}\left(0, T ; \mathbb{H}^{m}(\Omega)\right)} & \leq\left(\int_{0}^{T}\left|\varepsilon C(m, s) T\|F\|_{L^{\infty}\left(0, T ; \mathbb{H}^{s-1}(\Omega)\right)} t^{s-m-1}\right|^{\mu} d t\right)^{1 / \mu} \\
& =\varepsilon C(m, s) T\|F\|_{L^{\infty}\left(0, T ; \mathbb{H}^{s-1}(\Omega)\right)}\left(\frac{T^{(s-m-1) \mu+1}}{(s-m-1) \mu+1}\right)^{1 / \mu} .
\end{aligned}
$$

Combining (3.11), (3.12), (3.14), and (3.16), we find that

$$
\begin{aligned}
\left\|u_{\varepsilon}\right\|_{L^{\mu}\left(0, T ; \mathbb{H}^{m}(\Omega)\right)} & \leq\left\|I_{1}\right\|_{L^{\mu}\left(0, T ; \mathbb{H}^{m}(\Omega)\right)}+\left\|I_{2}\right\|_{L^{\mu}\left(0, T ; \mathbb{H}^{m}(\Omega)\right)}+\left\|I_{3}\right\|_{L^{\mu}\left(0, T ; \mathbb{H}^{m}(\Omega)\right)} \\
& \leq C(T, s, m, \mu)\left(\|f\|_{\mathbb{H}^{s}(\Omega)}+\|F\|_{\left.L^{\infty}\left(0, T ; \mathbb{H}^{s-1}(\Omega)\right)\right)} .\right.
\end{aligned}
$$

Let us recall the formula

$$
u(t)=\mathbf{S}(t) f+\int_{0}^{t}(t-r) \mathbf{S}(t-r) F(r) d r .
$$

Since (3.1), we get that

$$
u_{\varepsilon}(t)-u(t)=\left(\mathbf{Q}_{\varepsilon}(t)-\mathbf{S}(t)\right) f-\varepsilon \mathbf{Q}_{\varepsilon}(t) \int_{0}^{T}(T-r) \mathbf{S}(T-r) F(r) d r .
$$

From (3.16) we know that

$$
\begin{aligned}
& \left\|\varepsilon \mathbf{Q}_{\varepsilon}(t) \int_{0}^{T}(T-r) \mathbf{S}(T-r) F(r) d r\right\|_{L^{\mu}\left(0, T ; \mathbb{H}^{m}(\Omega)\right)} \\
& \quad \leq \varepsilon C(m, s) T\|F\|_{L^{\infty}\left(0, T ; \mathbb{H}^{s^{-1}}(\Omega)\right)}\left(\frac{T^{(s-m-1) \mu+1}}{(s-m-1) \mu+1}\right)^{1 / \mu} .
\end{aligned}
$$

Our next aim is estimating the term $\left(\mathbf{Q}_{\varepsilon}(t)-\mathbf{S}(t)\right) f$. We clearly see that

$$
\left(\mathbf{Q}_{\varepsilon}(t)-\mathbf{S}(t)\right) f=\sum_{n=1}^{\infty}\left[\frac{e^{-t \lambda_{n}}\left(1+t \lambda_{n}\right)}{1+\varepsilon e^{-T \lambda_{n}}\left(1+T \lambda_{n}\right)}-e^{-t \lambda_{n}}\left(1+t \lambda_{n}\right)\right]\left(\int_{\Omega} f(x) \psi_{n}(x) d x\right) \psi_{n}(x)
$$




$$
=\sum_{n=1}^{\infty}\left[\frac{\varepsilon e^{-T \lambda_{n}}\left(1+T \lambda_{n}\right) e^{-t \lambda_{n}}\left(1+t \lambda_{n}\right)}{1+\varepsilon e^{-T \lambda_{n}}\left(1+T \lambda_{n}\right)}\right]\left(\int_{\Omega} f(x) \psi_{n}(x) d x\right) \psi_{n}(x) .
$$

Parseval's equality implies that

$$
\begin{aligned}
& \left\|\left(\mathbf{Q}_{\varepsilon}(t)-\mathbf{S}(t)\right) f\right\|_{\mathbb{H}^{m}(\Omega)}^{2} \\
& =\sum_{n=1}^{\infty} \lambda_{n}^{2 m}\left[\frac{\varepsilon e^{-T \lambda_{n}}\left(1+T \lambda_{n}\right) e^{-t \lambda_{n}}\left(1+t \lambda_{n}\right)}{1+\varepsilon e^{-T \lambda_{n}}\left(1+T \lambda_{n}\right)}\right]^{2}\left(\int_{\Omega} f(x) \psi_{n}(x) d x\right)^{2} \\
& \leq \varepsilon^{2} \sum_{n=1}^{\infty} \lambda_{n}^{2 m} e^{-2 T \lambda_{n}}\left(1+T \lambda_{n}\right)^{2} e^{-2 t \lambda_{n}}\left(\int_{\Omega} f(x) \psi_{n}(x) d x\right)^{2} .
\end{aligned}
$$

Using the inequality $e^{-z} \leq C_{\nu} z^{-v}$ for all $v>0$, we arrive at

$$
e^{-2 t \lambda_{n}} \leq C(m, s)\left(t \lambda_{n}\right)^{-2(m+1-s)} .
$$

It is obvious that

$$
e^{-2 T \lambda_{n}}\left(1+T \lambda_{n}\right)^{2} \leq C
$$

It follows from (3.21) that

$$
\begin{aligned}
\left\|\left(\mathbf{Q}_{\varepsilon}(t)-\mathbf{S}(t)\right) f\right\|_{\mathbb{H}^{m}(\Omega)}^{2} & \leq C \varepsilon^{2} t^{2 m-2 s} \sum_{n=1}^{\infty} \lambda_{n}^{2 s-2}\left(\int_{\Omega} f(x) \psi_{n}(x) d x\right)^{2} \\
& =C(m, s) \varepsilon^{2} t^{2 s-2 m-2}\|f\|_{\mathbb{H}^{s-1}(\Omega)}^{2}
\end{aligned}
$$

This implies that

$$
\begin{aligned}
\left\|\left(\mathbf{Q}_{\varepsilon}-\mathbf{S}\right) f\right\|_{L^{\mu}\left(0, T ; \mathbb{H}^{m}(\Omega)\right)} & \leq C(m, s) \varepsilon\|f\|_{\mathbb{H}^{s-1}(\Omega)}\left(\int_{0}^{T} t^{(s-m-1) \mu} d t\right)^{1 / \mu} \\
& =C(m, s) \varepsilon\|f\|_{\mathbb{H}^{s-1}(\Omega)}\left(\frac{T^{(s-m-1) \mu+1}}{(s-m-1) \mu+1}\right)^{1 / \mu},
\end{aligned}
$$

where we recall that $1<\mu<\frac{1}{m+1-s}$. Combining (3.19), (3.20), and (3.24), we arrive at

$$
\begin{aligned}
& \left\|u_{\varepsilon}-u\right\|_{L^{\mu}\left(0, T ; \mathbb{H}^{m}(\Omega)\right)} \\
& \leq\left\|\left(\mathbf{Q}_{\varepsilon}-\mathbf{S}\right) f\right\|_{L^{\mu}\left(0, T ; \mathbb{H}^{m}(\Omega)\right)}+\left\|\varepsilon \mathbf{Q}_{\varepsilon}(t) \int_{0}^{T}(T-r) \mathbf{S}(T-r) F(r) d r\right\|_{L^{\mu}\left(0, T ; \mathbb{H}^{m}(\Omega)\right)} \\
& \leq C(m, s) \varepsilon\|f\|_{\mathbb{H}^{s-1}(\Omega)}\left(\frac{T^{(s-m-1) \mu+1}}{(s-m-1) \mu+1}\right)^{1 / \mu} \\
& \quad+\varepsilon C(m, s) T\|F\|_{L^{\infty}\left(0, T ; \mathbb{H}^{s-1}(\Omega)\right)}\left(\frac{T^{(s-m-1) \mu+1}}{(s-m-1) \mu+1}\right)^{1 / \mu} .
\end{aligned}
$$




\section{Well-posed results for nonlinear case}

Theorem 4.1 Let $f \in \mathbb{H}^{s}(\Omega)$ for $s \geq p$. Let $F$ be such that

$$
\|F(\varphi)-F(\psi)\|_{\mathbb{H}^{q}(\Omega)} \leq K_{f}\|\varphi-\psi\|_{\mathbb{H}^{p}(\Omega)}
$$

for all $\varphi, \psi \in \mathbb{H}^{p}(\Omega)$ and $p<q<p+1$. Then for any $\varepsilon>0$ and $K_{f}$ small enough, problem (1.1)-(1.2) has a unique mild solution in $\mathbf{X}^{a, \infty}\left((0, T] ; \mathbb{H}^{p}(\Omega)\right)$, which satisfies

$$
\begin{aligned}
u_{\varepsilon}(t)= & \mathbf{Q}_{\varepsilon}(t) f+\int_{0}^{t}(t-r) \mathbf{S}(t-r) F\left(u_{\varepsilon}(r)\right) d r \\
& -\varepsilon \mathbf{Q}_{\varepsilon}(t) \int_{0}^{T}(T-r) \mathbf{S}(T-r) F\left(u_{\varepsilon}(r)\right) d r,
\end{aligned}
$$

where

$$
\max (0, p+1-s) \leq a<1 .
$$

In addition,

$$
\left\|u_{\varepsilon}\right\|_{L^{\mu}\left(0, T ; \mathbb{H}^{p}(\Omega)\right)} \leq \frac{2 C_{T} T^{\frac{1}{\mu}+s-p-1}}{(1-a \mu)^{1 / \mu}}\|f\|_{\mathbb{H}^{s}(\Omega)}
$$

for $1<\mu<\frac{1}{a}$.

Proof We look for the solution in the space $\mathbf{X}^{a, \infty}\left((0, T] ; \mathbb{H}^{p}(\Omega)\right)$. Let us define the function

$$
\begin{aligned}
B_{\varepsilon}(\psi)(t)= & \mathbf{Q}_{\varepsilon}(t) f+\int_{0}^{t}(t-r) \mathbf{S}(t-r) F(\psi(r)) d r \\
& -\varepsilon \mathbf{Q}_{\varepsilon}(t) \int_{0}^{T}(T-r) \mathbf{S}(T-r) F(\psi(r)) d r .
\end{aligned}
$$

If $\psi=0$, then by the assumption $F(0)=0$ we have that

$$
t^{a}\left\|B_{\varepsilon} \psi(t)\right\|_{\mathbb{H}^{p}(\Omega)}=t^{a}\left\|\mathbf{Q}_{\varepsilon}(t) f\right\|_{\mathbb{H}^{p}(\Omega)} \leq C_{T} t^{a-v}\|f\|_{\mathbb{H}^{p+1-v}(\Omega)}
$$

Since $s<p+1$, we set $v=p+1-s$. Then it follows from (4.6) that

$$
t^{a}\left\|B_{\varepsilon} \psi(t)\right\|_{\mathbb{H}^{p}(\Omega)} \leq C_{T} t^{s+a-p-1}\|f\|_{\mathbb{H}^{s}(\Omega)}
$$

Under the assumption $p+1 \leq s+a$, if $\psi=0$, then we find that for any $0 \leq t \leq T$,

$$
t^{a}\left\|B_{\varepsilon}(\psi(t)=0)\right\|_{\mathbb{H}^{p}(\Omega)} \leq C_{T} T^{s+a-p-1}\|f\|_{\mathbb{H}^{s}(\Omega)},
$$

which allows us to derive that $B_{\varepsilon} \psi$ belongs to the space $\mathbf{X}^{a, \infty}\left((0, T] ; \mathbb{H}^{p}(\Omega)\right)$ if $\psi=0$.

Let $\varphi, \psi \in \mathbf{X}^{a, \infty}\left((0, T] ; \mathbb{H}^{p}(\Omega)\right)$. It is obvious that

$$
B_{\varepsilon}(\psi)(t)-B_{\varepsilon}(\varphi)(t)=\int_{0}^{t}(t-r) \mathbf{S}(t-r)(F(\psi(r))-F(\varphi(r))) d r
$$




$$
\begin{aligned}
& -\varepsilon \mathbf{Q}_{\varepsilon}(t) \int_{0}^{T}(T-r) \mathbf{S}(T-r)(F(\psi(r))-F(\varphi(r))) d r \\
= & J_{1}(t)+J_{2}(t) .
\end{aligned}
$$

By the second part of Lemma 3.1 the term $J_{1}$ is bounded by

$$
\begin{aligned}
\left\|J_{1}(t)\right\|_{\mathbb{H}^{p}(\Omega)} & \leq \int_{0}^{t}(t-r)\|\mathbf{S}(t-r)(F(\psi(r))-F(\varphi(r)))\|_{\mathbb{H}^{p}(\Omega)} d r \\
& \leq \int_{0}^{t}(t-r)(t-r)^{q-p}\|F(\psi(r))-F(\varphi(r))\|_{\mathbb{H}^{q}(\Omega)} d r
\end{aligned}
$$

where we note that $p>q$. Since $F$ is globally Lipschitz as in (4.1), we infer that

$$
\begin{aligned}
\left\|J_{1}(t)\right\|_{\mathbb{H}^{p}(\Omega)} & \leq K_{f} \int_{0}^{t}(t-r)^{q-p+1}\|\psi(r)-\varphi(r)\|_{\mathbb{H}^{p}(\Omega)} d r \\
& \leq K_{f}\left(\int_{0}^{t}(t-r)^{q-p+1} r^{-a} d r\right)\left(\sup _{0 \leq t \leq T} r^{a}\|\psi(r)-\varphi(r)\|_{\mathbb{H}^{p}(\Omega)}\right) \\
& =K_{f} B(2+q-p, 1-a) t^{2-a+q-p}\|\psi-\varphi\|_{\mathbf{X}^{a, \infty}\left((0, T] ; \mathbb{H}^{p}(\Omega)\right)},
\end{aligned}
$$

where we note that $q+2>p$ and $a<1$. This implies that

$$
\begin{aligned}
t^{a}\left\|J_{1}(t)\right\|_{\mathbb{H}^{p}(\Omega)} & \leq K_{f} B(2+q-p, 1-a) t^{2+q-p}\|\psi-\varphi\|_{\mathbf{X}^{a, \infty}\left((0, T] ; \mathbb{H}^{p}(\Omega)\right)} \\
& \leq K_{f} B(2+q-p, 1-a) T^{2+q-p}\|\psi-\varphi\|_{\mathbf{X}^{a, \infty}\left((0, T] ; \mathbb{H}^{p}(\Omega)\right)} .
\end{aligned}
$$

The right-hand side of this expression is independent of $t$, and we deduce that

$$
\left\|J_{1}\right\|_{\mathbf{X}^{a, \infty}\left((0, T] ; \mathbb{H}^{p}(\Omega)\right)} \leq K_{f} B(2+q-p, 1-a) T^{2+q-p}\|\psi-\varphi\|_{\mathbf{X}^{a, \infty}\left((0, T] ; \mathbb{H}^{p}(\Omega)\right)} .
$$

Since $q<p+1$ and $a>0$, we can choose a real number $s^{\prime}$ such that

$$
\max (p+1-a, q) \leq s^{\prime}<p+1
$$

Then we find that

$$
\begin{aligned}
& \left\|\mathbf{Q}_{\varepsilon}(t) \int_{0}^{T}(T-r) \mathbf{S}(T-r)(F(\psi(r))-F(\varphi(r))) d r\right\|_{\mathbb{H}^{p}(\Omega)} \\
& \quad \leq t^{s^{\prime}-p-1}\left\|\int_{0}^{T}(T-r) \mathbf{S}(T-r)(F(\psi(r))-F(\varphi(r))) d r\right\|_{\mathbb{H}^{s}(\Omega)} .
\end{aligned}
$$

Since $s^{\prime}>q$, we get that

$$
\begin{aligned}
& \left\|\int_{0}^{T}(T-r) \mathbf{S}(T-r)(F(\psi(r))-F(\varphi(r))) d r\right\|_{\mathbb{H}^{s}(\Omega)} \\
& \quad \leq \int_{0}^{T}(T-r)^{q-s^{\prime}+1}\|(F(\psi(r))-F(\varphi(r)))\|_{\mathbb{H}^{q}(\Omega)} d r
\end{aligned}
$$




$$
\begin{aligned}
& \leq K_{f} \int_{0}^{T}(T-r)^{q-s^{\prime}+1}\|\psi(r)-\varphi(r)\|_{\mathbb{H}^{p}(\Omega)} d r \\
& =K_{f} \int_{0}^{T}(T-r)^{q-s^{\prime}+1} r^{-a} r^{a}\|\psi(r)-\varphi(r)\|_{\mathbb{H}^{p}(\Omega)} d r \\
& \leq K_{f} B\left(2+q-s^{\prime}, 1-a\right) T^{2+q-s^{\prime}-a}\|\psi-\varphi\|_{\mathbf{X}^{a, \infty}\left((0, T] ; \mathbb{H}^{p}(\Omega)\right) .}
\end{aligned}
$$

Combining (4.14) and (4.15) and noting that $s^{\prime}+a \geq p+1$, we obtain that

$$
\begin{aligned}
t^{a}\left\|J_{2}(t)\right\|_{\mathbb{H}^{p}(\Omega)} & \leq \varepsilon t^{a+s^{\prime}-p-1} K_{f} B\left(2+q-s^{\prime}, 1-a\right) T^{2+q-s^{\prime}-a}\|\psi-\varphi\|_{\mathbf{X}^{a, \infty}\left((0, T] ; \mathbb{H}^{p}(\Omega)\right)} \\
& \leq \varepsilon K_{f} B\left(2+q-s^{\prime}, 1-a\right) T^{q+1-p}\|\psi-\varphi\|_{\mathbf{X}^{a, \infty}\left((0, T] ; \mathbb{H}^{p}(\Omega)\right)} .
\end{aligned}
$$

The condition $q+1>p$ ensures that the right-hand side is defined. Therefore we can deduce that

$$
\left\|J_{2}\right\|_{\mathbf{X}^{a, \infty}\left((0, T] ; \mathbb{H}^{p}(\Omega)\right)} \leq \varepsilon K_{f} B\left(2+q-s^{\prime}, 1-a\right) T^{q+1-p}\|\psi-\varphi\|_{\mathbf{X}^{a, \infty}\left((0, T] ; \mathbb{H}^{p}(\Omega)\right)}
$$

Combining (4.9), (4.13), and (4.17), we arrive at

$$
\begin{aligned}
&\left\|B_{\varepsilon}(\psi)-B_{\varepsilon}(\varphi)\right\|_{\mathbf{X}^{a, \infty}\left((0, T] ; \mathbb{H}^{p}(\Omega)\right)} \\
& \leq\left\|J_{1}\right\|_{\mathbf{X}^{a, \infty}\left((0, T] ; \mathbb{H}^{p}(\Omega)\right)}+\left\|J_{2}\right\|_{\mathbf{X}^{a, \infty}\left((0, T] ; \mathbb{H}^{p}(\Omega)\right)} \\
& \leq K_{f} B(2+q-p, 1-a) T^{2+q-p}\|\psi-\varphi\|_{\mathbf{X}^{a, \infty}\left((0, T] ; \mathbb{H}^{p}(\Omega)\right)} \\
&+\varepsilon K_{f} B\left(2+q-s^{\prime}, 1-a\right) T^{q+1-p}\|\psi-\varphi\|_{\mathbf{X}^{a, \infty}\left((0, T] ; \mathbb{H}^{p}(\Omega)\right)} .
\end{aligned}
$$

Let $K_{f}$ be small enough such that

$$
M_{T}=K_{f} B(2+q-p, 1-a) T^{2+q-p}+\varepsilon K_{f} B\left(2+q-s^{\prime}, 1-a\right) T^{q+1-p}<1 / 2 .
$$

It follows from (4.7) that

$$
B_{\varepsilon}\left(\mathbf{X}^{a, \infty}\left((0, T] ; \mathbb{H}^{p}(\Omega)\right)\right) \subset \mathbf{X}^{a, \infty}\left((0, T] ; \mathbb{H}^{p}(\Omega)\right),
$$

and together with (4.18), we find that $B_{\varepsilon}$ is a contraction mapping. By using the Banach fixed point theorem we deduce that roblem (1.1)-(1.2) has a unique solution $u_{\varepsilon} \in$ $\mathbf{X}^{a, \infty}\left((0, T] ; \mathbb{H}^{p}(\Omega)\right)$.

It follows from (4.8) that

$$
\begin{aligned}
\left\|u_{\varepsilon}\right\|_{\mathbf{X}^{a, \infty}\left((0, T] ; \mathbb{H}^{p}(\Omega)\right)} & =\left\|B_{\varepsilon}\left(u_{\varepsilon}\right)\right\|_{\mathbf{X}^{a, \infty}\left((0, T] ; \mathbb{H}^{p}(\Omega)\right)} \\
& \leq M_{T}\left\|u_{\varepsilon}\right\|_{\mathbf{X}^{a, \infty}\left((0, T] ; \mathbb{H}^{p}(\Omega)\right)}+C_{T} T^{s+a-p-1}\|f\|_{\mathbb{H}^{s}(\Omega)} .
\end{aligned}
$$

Therefore we get that

$$
\left\|u_{\varepsilon}\right\|_{\mathbf{X}^{a, \infty}\left((0, T] ; \mathbb{H}^{p}(\Omega)\right)} \leq \frac{C_{T} T^{s+a-p-1}\|f\|_{\mathbb{H}^{s}(\Omega)}}{1-M_{T}} \leq 2 C_{T} T^{s+a-p-1}\|f\|_{\mathbb{H}^{s}(\Omega)}
$$


This estimate implies that

$$
\left\|u_{\varepsilon}(t)\right\|_{\mathbb{H}^{p}(\Omega)} \leq 2 C_{T} T^{s+a-p-1} t^{-a}\|f\|_{\mathbb{H}^{s}(\Omega)}
$$

Since $a<1$, we can find that $0<\mu<\frac{1}{a}$. Thus we arrive at

$$
\begin{aligned}
\left\|u_{\varepsilon}\right\|_{L^{\mu}\left(0, T ; \mathbb{H}^{p}(\Omega)\right)} & =\left(\int_{0}^{T}\left\|u_{\varepsilon}(t)\right\|_{\mathbb{H}^{p}(\Omega)}^{\mu} d t\right)^{1 / \mu} \\
& \leq 2 C_{T} T^{s+a-p-1}\|f\|_{\mathbb{H}^{s}(\Omega)}\left(\int_{0}^{T} t^{-a \mu} d t\right)^{1 / \mu}
\end{aligned}
$$

which allows us to get that

$$
\left\|u_{\varepsilon}\right\|_{L^{\mu}\left(0, T ; \mathbb{H}^{p}(\Omega)\right)} \leq \frac{2 C_{T} T^{\frac{1}{\mu}+s-p-1}}{(1-a \mu)^{1 / \mu}}\|f\|_{\mathbb{H}^{s}(\Omega)} .
$$

The proof is completed.

Theorem 4.2 Let $F$ be as in (4.1). Let $f \in \mathbb{H}^{s}(\Omega)$ for $p<s<p+1$. Let $K_{f}$ be small enough such that $K_{f} T^{q-p+2} \leq \frac{1}{2}$. Then

$$
\begin{aligned}
\left\|u_{\varepsilon}-u\right\|_{L^{\mu}\left(0, T ; \mathbb{H}^{p}(\Omega)\right)} \leq & 2 C(p, s) \varepsilon\|f\|_{\mathbb{H}^{s-1}(\Omega)}\left(\frac{T^{(s-p-1) \mu+1}}{(s-p-1) \mu+1}\right)^{1 / \mu} \\
& +4 \varepsilon K_{f} T^{q-p}\|f\|_{\mathbb{H}^{s}(\Omega)}\left(\frac{T^{(s-p-1) \mu+1}}{(s-p-1) \mu+1}\right)^{1 / \mu}
\end{aligned}
$$

where $1<\mu<\frac{1}{p+1-s}$.

Proof Let us recall that

$$
u(t)=\bar{S}(t) f+\int_{0}^{t}(t-r) \mathbf{S}(t-r) F(u(r)) d r
$$

where we recall that

$$
\bar{S}(t) f=\sum_{n=1}^{\infty} e^{-t \lambda_{n}}\left(1+t \lambda_{n}\right)\left(\int_{\Omega} f(x) \psi_{n}(x) d x\right) \psi_{n}(x) .
$$

By (4.2) we immediately have the result on the difference between $u_{\varepsilon}(t)$ and $u(t)$ which is split as the sum of three terms

$$
\begin{aligned}
u_{\varepsilon}(t)-u(t)= & \mathbf{Q}_{\varepsilon}(t) f-\bar{S}(t) f+\int_{0}^{t}(t-r) \mathbf{S}(t-r)\left(F\left(u_{\varepsilon}(r)\right)-F(u(r))\right) d r \\
& -\varepsilon \mathbf{Q}_{\varepsilon}(t) \int_{0}^{T}(T-r) \mathbf{S}(T-r) F\left(u_{\varepsilon}(r)\right) d r \\
= & H_{1}(t)+H_{2}(t)+H_{3}(t) .
\end{aligned}
$$


Let us first treat the first term $H_{1}(t)$. By applying (3.24) we find that

$$
\begin{aligned}
\left\|\left(\mathbf{Q}_{\varepsilon}-\mathbf{S}\right) f\right\|_{L^{\mu}\left(0, T ; \mathbb{H}^{p}(\Omega)\right)} & \leq C(p, s) \varepsilon\|f\|_{\mathbb{H}^{s-1}(\Omega)}\left(\int_{0}^{T} t^{(s-p-1) \mu} d t\right)^{1 / \mu} \\
& =C(p, s) \varepsilon\|f\|_{\mathbb{H}^{s-1}(\Omega)}\left(\frac{T^{(s-p-1) \mu+1}}{(s-p-1) \mu+1}\right)^{1 / \mu}
\end{aligned}
$$

where we recall that $p+1>s>p$ and $1<\mu<\frac{1}{p+1-s}$.

The second term $H_{2}(t)$ by the second part of Lemma 3.1 is bounded by

$$
\begin{aligned}
\left\|H_{2}(t)\right\|_{\mathbb{H} p(\Omega)} & \leq \int_{0}^{t}(t-r)\left\|\mathbf{S}(t-r)\left(F\left(u_{\varepsilon}(r)\right)-F(u(r))\right)\right\|_{\mathbb{H} p(\Omega)} d r \\
& \leq \int_{0}^{t}(t-r)(t-r)^{q-p}\left\|F\left(u_{\varepsilon}(r)\right)-F(u(r))\right\|_{\mathbb{H} q(\Omega)} d r
\end{aligned}
$$

where we note that $p>q$. Since $F$ is globally Lipschitz as in (4.1), we infer that

$$
\begin{gathered}
\int_{0}^{t}(t-r)(t-r)^{q-p}\left\|F\left(u_{\varepsilon}(r)\right)-F(u(r))\right\|_{\mathbb{H}^{q}(\Omega)} d r \\
\quad \leq K_{f} \int_{0}^{t}(t-r)^{q-p+1}\left\|u_{\varepsilon}(r)-u(r)\right\|_{\mathbb{H}^{p}(\Omega)} d r .
\end{gathered}
$$

This implies that

$$
\begin{aligned}
\left\|H_{2}(t)\right\|_{\mathbb{H}^{p}(\Omega)} & \leq K_{f} \int_{0}^{t}(t-r)^{q-p+1}\left\|u_{\varepsilon}(r)-u(r)\right\|_{\mathbb{H}^{p}(\Omega)} d r \\
& \leq K_{f} T^{q-p+1} \int_{0}^{t}\left\|u_{\varepsilon}(r)-u(r)\right\|_{\mathbb{H}^{p}(\Omega)} d r \\
& \leq K_{f} T^{q-p+2}\left(\int_{0}^{t}\left\|u_{\varepsilon}(r)-u(r)\right\|_{\mathbb{H}^{p}(\Omega)}^{\mu} d r\right)^{1 / \mu} \\
& \leq K_{f} T^{q-p+2}\left\|u_{\varepsilon}-u\right\|_{L^{\mu}\left(0, T ; \mathbb{H}^{p}(\Omega)\right) .}
\end{aligned}
$$

Thus we obtain that

$$
\begin{aligned}
\left\|H_{2}\right\|_{L^{\mu}\left(0, T ; \mathbb{H}^{p}(\Omega)\right)} & \leq\left(\int_{0}^{T}\left(K_{f} T^{q-p+2}\left\|u_{\varepsilon}-u\right\|_{L^{\mu}\left(0, T ; \mathbb{H}^{p}(\Omega)\right)}\right)^{\mu}\right)^{1 / \mu} \\
& =K_{f} T^{q-p+2+\frac{1}{\mu}}\left\|u_{\varepsilon}-u\right\|_{L^{\mu}\left(0, T ; \mathbb{H}^{p}(\Omega)\right)} .
\end{aligned}
$$

For the third term $H_{3}(t)$, we apply Lemma 3.1 (noting that $s<p+1$ ) to get that

$$
\begin{gathered}
\left\|\mathbf{Q}_{\varepsilon}(t) \int_{0}^{T}(T-r) \mathbf{S}(T-r) F\left(u_{\varepsilon}(r)\right) d r\right\|_{\mathbb{H}^{p}(\Omega)} \\
\quad \leq t^{s-p-1}\left\|\int_{0}^{T}(T-r) \mathbf{S}(T-r) F\left(u_{\varepsilon}(r)\right) d r\right\|_{\mathbb{H}^{s}(\Omega)} .
\end{gathered}
$$


Since $s>q$, it follows from this estimate that

$$
\begin{aligned}
\left\|\int_{0}^{T}(T-r) \mathbf{S}(T-r) F\left(u_{\varepsilon}(r)\right) d r\right\|_{\mathbb{H}^{s}(\Omega)} & \leq \int_{0}^{T}(T-r)^{q-s+1}\left\|F\left(u_{\varepsilon}(r)\right)\right\|_{\mathbb{H}^{q}(\Omega)} d r \\
& \leq K_{f} \int_{0}^{T}(T-r)^{q-s+1}\left\|u_{\varepsilon}(r)\right\|_{\mathbb{H}^{p}(\Omega)} d r
\end{aligned}
$$

where in the last line, we have used that $F$ is globally Lipschitz. Recalling (4.21), we find that the right-hand side of (4.32) is bounded by

$$
\begin{aligned}
K_{f} & \int_{0}^{T}(T-r)^{q-s+1}\left\|u_{\varepsilon}(r)\right\|_{\mathbb{H}^{p}(\Omega)} d r \\
& \leq 2 K_{f} C_{T} T^{s+a-p-1}\|f\|_{\mathbb{H}^{s}(\Omega)} \int_{0}^{T}(T-r)^{q-s+1} r^{-a} d r \\
& =2 K_{f} C_{T} T^{s+a-p-1}\|f\|_{\mathbb{H}^{s}(\Omega)} T^{q-s+1-a} B(q-s+2,1-a)=2 K_{f} T^{q-p}\|f\|_{\mathbb{H}^{s}(\Omega)} .
\end{aligned}
$$

Combining (4.31), (4.32), and (4.33), we arrive at

$$
\begin{aligned}
\left\|H_{3}(t)\right\|_{\mathbb{H}^{p}(\Omega)} & \leq \varepsilon\left\|\int_{0}^{T}(T-r) \mathbf{S}(T-r) F\left(u_{\varepsilon}(r)\right) d r\right\|_{\mathbb{H}^{s}(\Omega)} \\
& \leq 2 \varepsilon K_{f} T^{q-p} t^{s-p-1}\|f\|_{\mathbb{H}^{s}(\Omega)} .
\end{aligned}
$$

This leads to

$$
\begin{aligned}
\left\|H_{3}\right\|_{L^{\mu}\left(0, T ; \mathbb{H}^{p}(\Omega)\right)} & \leq 2 \varepsilon K_{f} T^{q-p}\|f\|_{\mathbb{H}^{s}(\Omega)}\left(\int_{0}^{T} t^{(s-p-1) \mu} d t\right)^{1 / \mu} \\
& =2 \varepsilon K_{f} T^{q-p}\|f\|_{\mathbb{H}^{s}(\Omega)}\left(\frac{T^{(s-p-1) \mu+1}}{(s-p-1) \mu+1}\right)^{1 / \mu},
\end{aligned}
$$

where we recall that $p+1>s>p$ and $1<\mu<\frac{1}{p+1-s}$. Combining (4.26), (4.27), (4.30), and (4.35), we deduce that

$$
\begin{aligned}
\left\|u_{\varepsilon}-u\right\|_{L^{\mu}\left(0, T ; \mathbb{H}^{p}(\Omega)\right)} \leq & \sum_{j=1}^{3}\left\|H_{j}\right\|_{L^{\mu}\left(0, T ; \mathbb{H}^{p}(\Omega)\right)} \\
\leq & C(p, s) \varepsilon\|f\|_{\mathbb{H}^{s-1}(\Omega)}\left(\frac{T^{(s-p-1) \mu+1}}{(s-p-1) \mu+1}\right)^{1 / \mu} \\
& +2 \varepsilon K_{f} T^{q-p}\|f\|_{\mathbb{H}^{s}(\Omega)}\left(\frac{T^{(s-p-1) \mu+1}}{(s-p-1) \mu+1}\right)^{1 / \mu} \\
& +K_{f} T^{q-p+2}\left\|u_{\varepsilon}-u\right\|_{L^{\mu}\left(0, T ; \mathbb{H}^{p}(\Omega)\right) .}
\end{aligned}
$$

Let $K_{f}$ be small enough such that $K_{f} T^{q-p+2} \leq \frac{1}{2}$. Then from (4.36) the desired result follows. The proof is completed.

\section{Conclusion}

In this paper, we considered a biparabolic equation under temporal nonlocal conditions with linear and nonlinear source terms. We derived the regularity of the mild solution 
for the linear source term and applied the Banach fixed point theorem to study the existence and uniqueness of a mild solution for the nonlinear source term. In both cases, we demonstrated that the mild solution of our problem converges to the solution of an initial value problem as the parameter $\varepsilon \rightarrow 0$. The most compelling findings of our study can be considered as one of the first results on biparabolic equations with nonlocal conditions.

\section{Acknowledgements}

The authors would like to thank the supports from the National Research Foundation of Korea under grant number NRF-2020K1A3A1A05101625 and from the Institute of Construction and Environmental Engineering at Seoul National University. The authors also would like to thank the handling editor and two anonymous referees for their valuable and constructive comments to improve our manuscript.

\section{Funding}

This research was funded by the National Research Foundation of Korea under grant number NRF-2020K1A3A1A05101625 and the support from Institute of Construction and Environmental Engineering at Seoul National University.

\section{Availability of data and materials}

Not applicable.

\section{Competing interests}

The authors declare that they have no competing interests.

Authors' contributions

The authors contributed equally. All authors read and approved the final manuscript.

\section{Author details}

'Division of Applied Mathematics, Thu Dau Mot University, Thu Dau Mot, Binh Duong Province, Vietnam. ${ }^{2}$ Department of Civil and Environmental Engineering, Seoul National University, Seoul, South Korea.

\section{Publisher's Note}

Springer Nature remains neutral with regard to jurisdictional claims in published maps and institutional affiliations.

Received: 30 August 2021 Accepted: 22 September 2021 Published online: 01 October 2021

\section{References}

1. Adigüzel, R.S., Aksoy, U., Karapinar, E., Erhan, I.M.: On the solution of a boundary value problem associated with a fractional differential equation. Math. Methods Appl. Sci., 1-12 (2020). https://doi.org/10.1002/mma.6652

2. Adigüzel, R.S., Aksoy, U., Karapinar, E., Erhan, I.M.: On the solutions of fractional differential equations via Geraghty type hybrid contractions. Appl. Comput. Math. 20(2), 313-333 (2021)

3. Adigüzel, R.S., Aksoy, U., Karapinar, E., Erhan, I.M.: Uniqueness of solution for higher-order nonlinear fractional differential equations with multi-point and integral boundary conditions. Rev. R. Acad. Cienc. Exactas Fís. Nat., Ser. A Mat. 115(3), 155 (2021)

4. Alaoui, A.L., Azroul, E., Hamou, A.A.: Monotone iterative technique for nonlinear periodic time fractional parabolic problems. Adv. Theory Nonlinear Anal. Appl. 4(3), 194-213 (2020) 2020

5. Besma, K., Nadji, B., Faouzia, R.: A modified quasi-boundary value method for an abstract ill-posed biparabolic problem. Open Math. 15, 1649-1666 (2017)

6. Bulavatsky, V.M.: Mathematical modeling of filtrational consolidation of soil under motion of saline solutions on the basis of biparabolic model. J. Autom. Inf. Sci. 35(8), 13-22 (2003)

7. Bulavatsky, V.M.: Fractional differential analog of biparabolic evolution equation and some its applications. Cybern. Syst. Anal. 52(5), 337-347 (2016)

8. Bulavatsky, V.M., Skopetsky, V.V.: Generalized mathematical model of the dynamics of consolidation processes with relaxation. Cybern. Syst. Anal. 44(5), 646-654 (2008)

9. Fushchich, V.L., Galitsyn, A.S., Polubinskii, A.S.: A new mathematical model of heat conduction processes. Ukr. Math. J. 42, 210-216 (1990)

10. Georgiev, S.G., Zennir, K.: New results on IBVP for class of nonlinear parabolic equations. Adv. Theory Nonlinear Anal. Appl. 2(4), 202-216 (2018)

11. Halpern, D., Grotberg, J.B.: Dynamics and transport of a localized soluble surfactant on a thin film. J. Fluid Mech. 237, 1-11 (1992)

12. Halpern, D., Jensen, O.E., Grotberg, J.B.: A theoretical study of surfactant and liquid delivery into the lung. J. Appl. Physiol. 85, 333-352 (1998)

13. Hammou, M.A., Azroul, E.: Existence of weak solution for a nonlinear parabolic equations by topological degree. Adv. Theory Nonlinear Anal. Appl. 4(4), 292-298 (2020)

14. Kalantarov, V., Zelik, S.: Finite-dimensional attractors for the quasi-linear strongly-damped wave equation. J. Differ. Equ. 247(4), 1120-1155 (2009)

15. Karapinar, E., Binh, H.D., Luc, N.H.: On continuity of the fractional derivative of the time-fractional semilinear pseudo-parabolic systems. Adv. Differ. Equ. 2021, 70 (2021) 
16. Lakhdari, A., Boussetila, N.: An iterative regularization method for an abstract ill-posed biparabolic problem. Bound. Value Probl. 2015, 55, 1-17 (2015)

17. Luc, N.H., Long, L.D., Van, H.T.K., Nguyen, V.T: A nonlinear fractional Rayleigh-Stokes equation under nonlocal integral conditions. Adv. Differ. Equ. 2021, 388 (2021)

18. Memoli, F., Sapiro, G., Thompson, P.: Implicit brain imaging. Hum. Brain Mapp. 23, 179-188 (2004)

19. Myers, T.G.: Extension to the Messinger model for aircraft icing. AIAA J. 39(2), 211-218 (2001)

20. Myers, T.G., Charpin, J.P.F.: A mathematical model for atmospheric ice accretion and water flow on a cold surface. Int. J. Heat Mass Transf. 47(25), 5483-5500 (2004)

21. Myers, T.G., Charpin, J.P.F., Chapman, S.J.: The flow and solidification of a thin fluid film on an arbitrary three-dimensional surface. Phys. Fluids 14(8), 2788-2803 (2002)

22. Nam, D.H.Q., Baleanu, D., Luc, N.H., Can, N.H.: On a Kirchhoff diffusion equation with integral condition. Adv. Differ Equ. 2020(1), 617, 1-15 (2020)

23. Pata, V., Squassina, M.: On the strongly damped wave equation. Commun. Math. Phys. 253(3), 511-533 (2005)

24. Payne, L.E.: On a proposed model for heat conduction. IMA J. Appl. Math. 71, 590-599 (2006)

25. Phuong, N.D., Luc, N.H., Long, L.D.: Modified quasi boundary value method for inverse source problem of the bi-parabolic equation. Adv. Theory Nonlinear Anal. Appl. 4(3), 132-142 (2020). https://doi.org/10.31197/atnaa.752335

26. Thompson, P., Toga, A.W.: A surface-based technique for warping three-dimensional images of the brain. IEEE Trans. Med. Imaging 15(4), 402-417 (1996)

27. Toga, A.: Brain Warping. Academic Press, New York (1998)

28. Triet, N.A., Binh, T.T., Phuong, N.D., Baleanu, D., Can, N.H.: Recovering the initial value for a system of nonlocal diffusion equations with random noise on the measurements. Math. Methods Appl. Sci. 44(6), 5188-5209 (2021)

29. Tuan, N.A., Hammouch, Z., Karapinar, E., Tuan, N.H.: On a nonlocal problem for a Caputo time-fractional pseudoparabolic equation. Math. Methods Appl. Sci. (2021). https://doi.org/10.1002/mma.7743

30. Tuan, N.A., O'Regan, D., Baleanu, D., Tuan, N.H.: On time fractional pseudo-parabolic equations with nonlocal integral conditions. Evol. Equ. Control Theory (2020). https://doi.org/10.3934/eect.2020109

31. Tuan, N.H., Caraballo, T., Thach, T.N.: On terminal value problems for bi-parabolic equations driven by Wiener process and fractional Brownian motions. Asymptot. Anal. 123(3-4), 335-366 (2021)

32. Tuan, N.H., Kirane, M., Nam, D.H.Q., Au, V.V.: Approximation of an inverse initial problem for a biparabolic equation. Mediterr. J. Math. 15, 18, 18 pages (2018)

33. Wang, L., Zhou, X., Wei, X.: Heat Conduction: Mathematical Models and Analytical Solutions. Springer, Berlin (2008)

34. Yang, Y., Salik Ahmed, M., Qin, L., Xu, R.: Global well-posedness of a class of fourth-order strongly damped nonlinear wave equations. Opusc. Math. 39(2), 297-313 (2019)

\section{Submit your manuscript to a SpringerOpen ${ }^{\circ}$ journal and benefit from:}

- Convenient online submission

- Rigorous peer review

- Open access: articles freely available online

- High visibility within the field

- Retaining the copyright to your article

Submit your next manuscript at $\gg$ springeropen.com 